Regulatory review:

Experts ponder

impact of UK law

on embryo research

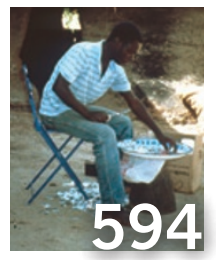

Neglected disease:

The fight against

parasitic illnesses

gains momentum

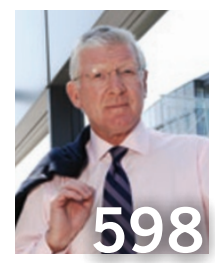

Cash injection:

Alan Gillepsie talks

about a novel way to

fund vaccines

\title{
Concerns over 'shifting sands' linger as genetic testing expands
}

With the increased availability of personal genetic testing, it has become far easier to 'know thyself', as the ancient Greek aphorism recommends. Individuals can gain unprecedented insight into their DNA by simply sending off a vial of their saliva and paying a fee for its analysis. Still, even as new companies move into the market offering such services, some scientists caution that these tests might offer an inexact picture of one's genetic predisposition to disease.

In the US the focus on personal genetic testing has increased in large part owing to lawmakers' approval this spring of the long stalled Genetic Information Nondiscrimination Act. This measure - signed into law by President George W. Bush on 21 May-prohibits health insurance companies from denying coverage on the basis of an applicant's personal genetic data.

This legal protection should make US consumers more comfortable with the idea of purchasing personal genetic tests, and the range of options for such tests continues to grow. In the past, genetic tests have typically provided information about rare inherited disorders after close scrutiny of a specific gene. But, within the past year, several companies have launched testing services that help estimate one's risk of developing various common illnesses, such as heart disease, based on a broad view of the genome. For companies such as deCODEme, 23andMe and Navigenics, which offer risk information about common diseases, that means analyzing between 500,000 to 1 million points across the genome called single nucleotide polymorphisms (SNPs).

What concerns some experts is that these tests do not yet capture all the genetic risk markers for disease, meaning that the estimates made by the tests will probably change as new scientific findings emerge: "The ground that we're standing on is shifting sands," says Colleen McBride, who heads the Public Health Genomics Section at the US National Human Genome Research Institute in Bethesda, Maryland.

Scientists note that the statistical support for disease-SNP associations has improved markedly in recent years. But they add that some uncertainty remains around the biological effects of the individual genetic variations analyzed. "We have definitely seen a lot of progress in the field in the past couple of years," says John Ioannidis of the University of Ioannina School of Medicine in Ioannina, Greece. "The caveats that remain are that these effects we've identified are small effects."

"These markers are all interesting, but individually they're very weak markers," says Michael Watson, executive director of the American College of Medical Genetics (ACMG) in Bethesda. "The health care system does not think it's time yet for many of these tests." In late April, the ACMG also called for tighter government regulation of the laboratories conducting these tests to ensure accurate reading of DNA samples.

The possibility remains that genetic tests might falsely alarm (or falsely reassure) people who take them. For example, in April 2006, researchers identified a common genetic variant near the INSIG2 gene and estimated that individuals with two copies of the variant were 22\% more likely to become obese (Science 312, 279-283; 2006). But more recent analyses have found the opposite or have failed to replicate this association in normal-weight individuals (Science 315, 187; 2007).

\section{Watching the numbers}

Ioannidis says that study designs have improved in the past couple of years to avoid mistaken associations. However, he adds that for complex statistical reasons, the associations identified by current trials might still be slightly inflated.

Meanwhile, Thomas Morgan of the Washington University School of Medicine in St. Louis, Missouri cautions that customers receiving information about their genetic risk of common diseases might not grasp that their lifestyle and ancestry influence their reported risk relative to the best published evidence. He likens the practice of returning test results without appropriate counseling to "dumping a pile of laboratory notebooks on people and asking them to make sense of it all. A glossy brochure, even if it's really well written, cannot put this information into context the way a doctor can."

Companies offering personal genetic testing counter that they offer services that provide an

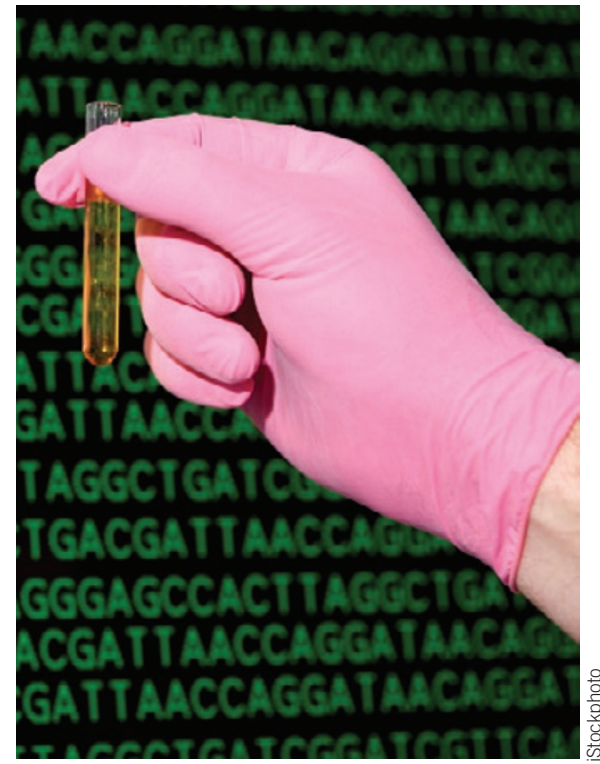

Known unknowns: Untested gene regions count

interactive and informative means of conveying results. Navigenics, for example, offers overthe-phone genetic counseling as part of its testing package. Elissa Levin, director of genetic counseling for Navigenics in Redwood Shores, California, says this allows the company to answer customers' questions about their results as they come up: "It's really critical that people understand this is a snapshot and that there is new information that is emerging."

To ensure the scientific accuracy of the test results given to customers, Navigenics includes information about each person's lifestyle and age in calculating individual disease risk. And, among other requirements, the company relies only on genetic studies of disease that include at least 250 cases and 250 controls.

23andMe uses an approach in which its experts rate genetic associations with stars according to the scientific evidence backing them up, with four stars as the highest score. As customers scroll through their results on the website, they can view these ratings. "We're pretty stringent [about] what gets to be fourstar," says Linda Avey, who co-founded the Mountain View, California-based company.

Roxanne Khamsi, New York 